\title{
A Topological Investigation of the Quantum Adiabatic Phase*
}

\author{
Elias Kiritsis \\ California Institute of Technology, Pasadena, CA 91125, USA
}

\begin{abstract}
Using algebraic topology, the appearance of the Quantum Adiabatic Phase over various parameter manifolds is investigated. The relation with nontrivial gauge bundles (both abelian and non-abelian) is studied and it is shown that the phase appears as a result of homotopically non-trivial mappings, induced by the Hamiltonian in the space of wave-functions. The cohomological picture is developed and some topological considerations concerning field theory anomalies in the Hamiltonian picture are presented. A proof of the Nielsen-Ninomiya theorem is given inspired from the notion of the adiabatic phase.
\end{abstract}

\section{Introduction}

Recently, M. Berry found that the usual form of the Quantum Adiabatic Theorem was not exactly correct, [1]. He showed that when the Hamiltonian depends on several parameters that change adiabatically with time and the topology of the manifold spanned by the parameters is non-trivial (usually induced by accidental degeneracies), there is another contribution to the phase acquired by the wavefunction of the system (under adiabatic transport). This is topological in nature and it is related to the first Chern class of the natural hermitian (abelian) connection in the Hilbert bundle over the parameter manifold [2].

Later, Wilczek and Zee, [3], generalized the notion of the adiabatic phase to the non-abelian case corresponding to adiabatically transporting an $n$-fold degenerate state over the entire parameter manifold.

This discovery turned out to be very fruitful in a lot of situations, including applications to diatoms [4], modifications of the Bohr-Sommerfeld semiclassical quantization in some special cases [5], the explanation of the quantum Hall effect

\footnotetext{
* Work supported in part by the U.S. Department of Energy under contract DEAC 03-81-
} ER 40050 
$[13,14]$, and an appealing interpretation of gauge anomalies (in the Hamiltonian picture) $[6,7]$, as well as the successful calculation of anomalous commutators in chiral gauge theories $[8,9]$.

The motivation for the present work is the following: since gauge structures appear as a result of adiabatic transport over various parameter spaces, we can classify the non-trivial Quantum Adiabatic Phases by topologically classifying the respective gauge bundles.

It turns out that in the most interesting cases the classification of the Berry phase is equivalent to classifying gauge bundles over spheres. The topology of the sphere is induced in the parameter space by degeneracies (level crossings). The picture involving level crossings gives an appealing intuitive way to understand the global obstructions responsible for the adiabatic phase. There are situations though (outside the stable range, in most cases) where not every non-trivial gauge bundle can appear as a result of adiabatic transport. Some examples are discussed in the main body of this paper. These non-trivial gauge bundles are characterized by their Chern classes, built out of the curvature 2-form $F$, which generates the "Quantum Holonomy". The Chern classes above when integrated over the parameter manifold give integers associated with the winding number of the map, (induced by the Hamiltonian), from the parameter space to the appropriate space of wave functions.

The structure of this paper is as follows: In Sect. 2 we review the correct form of the Quantum Adiabatic Theorem following [1,2]. In Sect. 3 we identify the global obstructions to smoothly defining the phase of the wave-function on the parameter manifold and present a theorem that classifies completely the existence of a topologically unremovable phase over a compact manifold. In Sect. 4 we discuss the situation of transporting degenerate levels (non-abelian structure relevant here) and in Sect. 5 we identify the obstructions to the global definition of the phase as nontrivial Chern classes of the parameter manifold generated by the gauge connection of the appropriate Hilbert bundle. In Sect. 6 gauge anomalies in the Hamiltonian picture are revisited as obstructions to defining the phase of a gauge invariant vacuum state over the manifold of gauge transformations. In Sect. 7 we present a proof of the Nielsen-Ninomiya theorem, [12], inspired from the notion of the Quantum Adiabatic Phase. Finally Sect. 8 contains the conclusions.

In Appendix A some pertinent results on the homotopy theory of fibre bundles and obstruction theory are presented. In Appendix B we prove the theorem used in Sect. 3 to classify the obstructions that lead to an irremovable adiabatic phase. In Appendix $\mathrm{C}$ we examine some examples illustrating how to trace the possible existence of a non-trivial phase.

\section{Review of the Quantum Adiabatic Theorem}

Suppose that we have a hermitian Hamiltonian $H(t)$ which depends on time through some parameters $\lambda_{i}(t) i=1,2, \ldots, n$ that parametrize some manifold $M$.

We will work in the adiabatic approximation, which means that the change of these parameters with time is slow enough so that if the system was originally in some eigenstate of the Hamiltonian, it will continue to remain in that state. 
The Schrödinger equation is:

$$
i \frac{\partial}{\partial t} \psi=\hat{H}(\vec{\lambda}(t)) \psi
$$

at any instant, which means that at each point of $M$ we can choose an orthonormal basis that diagonalizes the hamiltonian.

$$
\hat{H}(\vec{\lambda})|n(\vec{\lambda})\rangle=E_{n}(\vec{\lambda})|n(\vec{\lambda})\rangle .
$$

The adiabatic approximation states that if $|\psi(0)\rangle=|n(\vec{\lambda}(0))\rangle$, then $|\psi(t)\rangle$ $=|n(\vec{\lambda}(t))\rangle$ up to a phase. To find the phase acquired by the wave-function under adiabatic transport we will separate the dynamical phase from anything "else":

$$
|\psi(t)\rangle=\exp \left\{-i \int_{0}^{t} d t^{\prime} E_{n}\left(\vec{\lambda}\left(t^{\prime}\right)\right)\right\} e^{i \gamma_{n}(t)}|n(\vec{\lambda}(t))\rangle .
$$

Substituting this form in (2.1) we find an equation for $\gamma_{n}(t)$ :

$$
\frac{d}{d t} \gamma_{n}(t)=i\left\langle n(\vec{\lambda}(t))\left|\vec{\nabla}_{\vec{\lambda}}\right| n(\vec{\lambda}(t))\right\rangle \cdot \frac{d \vec{\lambda}}{d t} .
$$

Suppose we trace a closed contour $C$ in $M$. Then solving (2.4) we get:

$$
\gamma_{n}=i \int_{C}\langle n(\vec{\lambda})|\vec{\nabla} \vec{\lambda}| n(\vec{\lambda})\rangle \cdot d \vec{\lambda}
$$

which is the additional contribution to the adiabatic phase.

Defining

$$
\vec{A}=\langle n(\vec{\lambda})|\vec{\nabla} \vec{\lambda}| n(\vec{\lambda})\rangle
$$

it is easy to realize that $\vec{A}$ is a connection in the $n^{\text {th }}$ level Hilbert subbundle and for this connection the Schrödinger equation is the equation of parallel transport. In this sense this connection is natural.

Using the language of forms we can write:

$$
A=\langle n(\vec{\lambda}) \mid d n(\vec{\lambda})\rangle, \quad F=d A, \quad \gamma_{n}=\int_{S} F
$$

where $S$ is a surface that has $C$ as boundary. In the above form the extra phase is given as an integral of the first Chern class of the connection and thus is of a topological origin [2].

Berry, [1], also pointed out the importance of accidental degeneracies on $M$ which are essential for the existence of a non-trivial topological phase. Thus, the adiabatic phase is due to the non-trivial topology of the parameter manifold, induced by degeneracies, which obstruct the smooth definition of the phase of the transported wave-function.

In the following we will renormalize the Hamiltonians so that the adiabatically transported state has zero energy over $M$, assuring in this way that there is no dynamical phase.

There is a simple example which shows all the important features of the adiabatic phase and also gives hints for a general topological classification of 
parameter manifolds that will cause such a phase to appear. We will consider a two level system described by a two-by-two hermitian hamiltonian matrix depending on three parameters assembled in a $3-d$ vector $\vec{x}$. Suppose that at $\vec{x}_{0}$ the two eigenvalues are degenerate, but if we depart from $\vec{x}_{0}$ in any direction the degeneracy disappears. Near the degeneracy point we can write to first-order in $\vec{x}-\vec{x}_{0}$ :

$$
\hat{H}(\vec{x})=\hat{H}\left(\vec{x}_{0}\right)+\sigma^{i} C_{i j}\left(\vec{x}-\vec{x}_{0}\right)^{j}+\vec{B} \cdot\left(\vec{x}-\vec{x}_{0}\right)+O\left(\left|\vec{x}-\vec{x}_{0}\right|^{2}\right),
$$

where $\sigma^{i}$ are the standard Pauli matrices and $C_{i j}$ is a non-singular matrix. Renormalizing the energy such that $\hat{H}\left(\vec{x}_{0}\right)=0$ and shifting coordinates such that $\vec{x}_{0}=\overrightarrow{0}$ :

$$
\hat{H}(\vec{x})=B_{i} x^{i}+C_{i j} x^{i} \sigma^{j}+O\left(\vec{x}^{2}\right) .
$$

Now it is easy to calculate the adiabatic phase in this case: Changing coordinates, $p_{i}=C_{i j} x^{j}$, and using (2.6) we can compute the "magnetic field" corresponding to $\vec{A}$ :

$$
\vec{B}_{ \pm}=\mp \operatorname{sign}(\operatorname{det} C) \frac{\vec{p}}{2 p^{3}}
$$

where \pm labels the two levels, and

$$
\gamma_{ \pm}= \pm \operatorname{sign}(\operatorname{det} C) \int_{S} \frac{d S}{2 p^{2}}= \pm \frac{1}{2} \operatorname{sign}(\operatorname{det} C) \Omega_{s},
$$

where $\Omega_{s}$ is the solid angle subtend by the contour $C$ as seen from the degeneracy point, $\vec{x}=\overrightarrow{0}$. In particular for the contour shown in Fig. 1:

$$
\gamma_{ \pm}= \pm \operatorname{sign}(\operatorname{det} C) \pi
$$

and both wave-functions pick up a minus sign after coming back to the initial point of the parameter manifold (which has the topology of a 2-sphere due to the degeneracy point at $\vec{x}=0$ ).

Consider a sphere of unit radius surrounding the degeneracy. The eigenstates of the Hamiltonian can be written in spherical coordinates:

$$
u_{+}=\left[\begin{array}{c}
\cos \frac{\theta}{2} e^{-i \phi} \\
\sin \frac{\theta}{2}
\end{array}\right] \text {, }
$$

Fig. 1

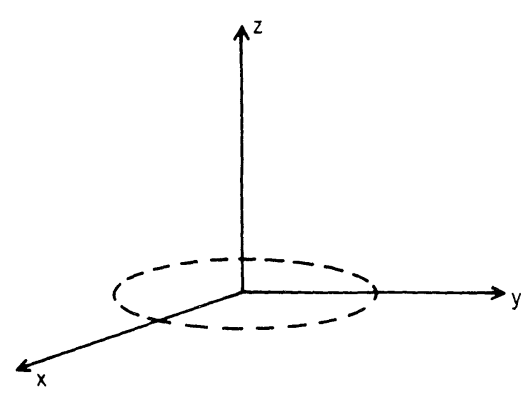




$$
u_{-}=\left[\begin{array}{c}
-\sin \frac{\theta}{2} e^{-i \phi} \\
\cos \frac{\theta}{2}
\end{array}\right]
$$

It is obvious that the phase of $u_{+}$is ill-defined at the North-pole (respectively at the South-pole for $u_{-}$), or that the mapping from the 2-sphere to $\mathbf{C}^{2}$ is $1-1$ but not well defined at the North (South)-pole. The $U(1)$ gauge potentials are:

$$
\begin{aligned}
& \vec{A}_{+}=\frac{1}{2} \cot \frac{\theta}{2} \hat{e}_{\phi}, \\
& \vec{A}_{-}=\frac{1}{2} \tan \frac{\theta}{2} \hat{e}_{\phi},
\end{aligned}
$$

and they have a string singularity going through the North $(+)$ or the South $(-)$ pole. Multiplying the wave-functions with a phase induces a $U(1)$ gauge transformation on $\vec{A}$, thus moving the singularity around the sphere. The above gauge connection is the same as that of a magnetic monopole of strength one-half situated at the origin.

The curvature form $F$ though (the magnetic field), is well defined everywhere (with the exception of the origin). In this case it is apparent that cohomology plays an important role; we have a closed $(d F=0)$ but not exact 2-form (due to the singular nature of the gauge potential). As we will see in the sequel this feature persists in the more general case.

The adiabatic phase in this case arises due to the impossibility of defining smoothly and globally the phase of the adiabatically transported wave-functions of the Hamiltonian over the parameter manifold (which is a two-sphere).

The above results are generalized in the case where a state is $n$-fold degenerate over the entire parameter manifold $M$, [3]. Then an adiabatic transport around a closed curve in $M$ can induce a $U(n)$ rotation of the $n$ degenerate levels.

\section{Topological Classification of the Adiabatic Phase}

In this section we will formulate the problem in mathematical terms using the formalism of fibre bundles and obstruction theory.

We are interested in topologically irremovable adiabatic phases because in the opposite case we can redefine them away by redefining the Hamiltonian and the states in the Hilbert space.

Let's consider the simplest example of a two-level Hamiltonian, to give a feeling about the general situation. The wave-functions in this case are two-dimensional complex vectors. As in the previous section the Hamiltonian depends on certain parameters that parametrize a compact manifold $M$. The adiabatic transport of an eigenstate of the Hamiltonian defines a mapping from a submanifold of $M$ to the space of two-dimensional complex vectors of unit modulus, up to a phase ( $\left.\equiv \mathbf{C P}^{1}\right)$. The space of two-dimensional complex vectors of unit modulus is topologically equivalent to $S^{3}$. We will suppose for the time being that the state in question is not 
degenerate with any other state over the entire parameter manifold (but there may be accidental degeneracies).

In this case the Hamiltonian defines a mapping $f: M \rightarrow \mathbf{C P}^{1}$. If a global definition of the phase of the wave-function is impossible over $M$ then adiabatic transport will give a Berry phase. So the question of appearance of a non-trivial adiabatic phase is equivalent in this case to the possibility (or not) of lifting $f: M \rightarrow \mathbf{C P}^{1}$ to $\widetilde{f}: M \rightarrow \mathbf{S}^{3}$. Then, since $\mathbf{S}^{3}$ is a deformation retraction of $\mathbf{C}^{2}$, the global definition of the phase is possible if and only if such a lifting $\tilde{f}$ exists.

There is a relevant fibration in this case (Hopf fibration), which is crucial in deciding about the possibility of lifting $f$.

$$
\mathbf{U}(1) \stackrel{i}{\rightarrow} \mathbf{S}^{3} \stackrel{p}{\rightarrow} \mathbf{C} \mathbf{P}^{1} \text {. }
$$

An example of a mapping that cannot be lifted when $M=\mathbf{S}^{2}$ is one belonging to a non-trivial class of $\pi_{2}\left(\mathbf{C} \mathbf{P}^{1}\right)=\mathbf{Z}$. If such a mapping could be lifted, then since $\pi_{2}\left(\mathbf{S}^{3}\right)=0$ there would be a homotopy $h$ such that $h(0)=\widetilde{f}, h(1)=\widetilde{f}_{0}$ the trivial map. Then $p \circ h$ is a homotopy connecting $f$ to the trivial map $p \circ \widetilde{f}_{0}: \mathbf{S}^{2} \rightarrow \mathbf{C P} \mathbf{P}^{1}$ which is false by assumption.

In the general case of adiabatic transport of a single non-degenerate level of an $N \times N$ hermitian Hamiltonian, the wave-functions are vectors in $\mathbf{C}^{N}$ and the Hamiltonian induces maps $f: M \rightarrow \mathbf{C} \mathbf{P}^{N-1}$, so that the relevant question in this case is the possibility of lifting $f$ to $\widetilde{f}, p \circ \widetilde{f}=f$.

To classify the possible obstructions we will make use of the following theorem (the proof is given in Appendix B).

Consider a general principal fiber bundle $B \equiv(\mathbf{B}, \mathbf{A}, \mathbf{F})$, where $\mathbf{B}$ is the base space, $\mathbf{A}$ is the bundle space, $\mathbf{F}$ is the fibre and at the same time the structure group of the bundle and a manifold $M$ along with a mapping $f: M \rightarrow \mathbf{B}$,

$$
M \stackrel{f}{\rightarrow} \mathbf{B} \stackrel{p}{\leftarrow} \mathbf{A} \stackrel{i}{\leftarrow} \mathbf{F} .
$$

We will try to find the condition for the existence of the lifting $\widetilde{f}$ of $f,(p \circ \tilde{f}=f)$ $\tilde{f}: M \rightarrow \mathbf{A}$. The projection $p$ induces a mapping $p^{1}:[M, \mathbf{A}] \rightarrow[M, \mathbf{B}]([M, \mathbf{B}]$ is the set of homotopy classes of mappings from $M$ to $\mathbf{B}$. In all of our applications it will have the structure of an abelian group.)

Theorem. $f$ can be lifted if and only if it belongs to a class in $\operatorname{Im} p^{*}$, the image of $[M, \mathbf{A}]$ under $p^{* 1}$.

The information concerning $\operatorname{Im} p^{*}$ can in general be found by analyzing the exact homotopy sequence of the respective fibration.

A comment is in place here. The possibility to lift a map $f: M \rightarrow \mathbf{B}$ to $\tilde{f}=M \rightarrow \mathbf{A}$ is equivalent to the ability of finding a global cross-section in the cofibration over $M$ induced by the pullback of $f$. If such a lifting exists, the pullback bundle is a trivial one. So, the classification of mappings that cannot be lifted is equivalent to classifying all non-trivial bundles over $M$ which are the pullbacks of $B$. These obviously are a subset of all possible non-trivial bundles over $M$ (having the same fibre as $B$ ).

\footnotetext{
${ }^{1}$ For a proof see Appendix B
} 
Let's now analyze our first case pertinent to a single non-degenerate level. The structure in this case is:

$$
M \stackrel{f}{\rightarrow} \mathbf{C P}^{N-1} \stackrel{p}{\leftarrow} \mathbf{S}^{2 N-1} \stackrel{i}{\leftarrow} \mathbf{U}(1),
$$

where $M=\mathbf{S}^{n}$ for some $n \geqq 1$. Using the exact sequence of the bundle $\left(\pi_{n}(\mathbf{U}(1)) \approx 0\right.$ for $n=0$ or $\left.n \geqq 2, \pi_{1}(\mathbf{U}(1)) \approx \mathbf{Z}\right)$

$$
0 \stackrel{i^{*}}{\rightarrow} \pi_{n}\left(\mathbf{S}^{2 N-1}\right) \stackrel{p^{*}}{\rightarrow} \pi_{n}\left(\mathbf{C} \mathbf{P}^{N-1}\right) \stackrel{\partial}{\rightarrow} 0 \text { for } n \geqq 3,
$$

which means that $p^{*}$ is onto and consequently there is no obstruction in the global definition of phase over parameter manifolds $\mathbf{S}^{n}$ with $n \geqq 3$. For $n=2$ and $N>1: \pi_{1}\left(\mathbf{S}^{2 N-1}\right) \approx 0$ and $\pi_{1}\left(\mathbf{C} \mathbf{P}^{N-1}\right) \approx 0$, whereas $\pi_{2}\left(\mathbf{S}^{2 N-1}\right) \approx 0$ and $\pi_{2}\left(\mathbf{C} \mathbf{P}^{N-1}\right) \approx \mathbf{Z}$. So there is no obstruction on $\mathbf{S}^{1}$ but for $M=\mathbf{S}^{2}$ if the induced mapping belongs to a non-trivial class of $\pi_{2}\left(\mathbf{C} \mathbf{P}^{N-1}\right)$ then an adiabatic phase will appear. This corresponds exactly to the situation of the example discussed in Sect. 2. The phase in this case is due to the non-triviality of $\pi_{2}\left(\mathbf{C} \mathbf{P}^{N-1}\right)$. (This remark helps in providing an easy alternative proof of the Nielsen-Ninomiya theorem, see Sect. 7.)

The situation above is alternatively understood as follows: The first Chern class $F$, which after integration over a surface gives the adiabatic phase, is the winding number density of $\pi_{2}\left(\mathbf{C} \mathbf{P}^{N-1}\right)$. In particular the integral of $F$ over $\mathbf{S}^{2}$ gives $2 \pi \times$ (the winding number of the map) $|n\rangle: \mathbf{S}^{2} \rightarrow \mathbf{C} \mathbf{P}^{N-1}$. This also shows that to calculate the phase picked-up after a diabatic transport around a closed contour $C$ we can choose either $S_{1}$ or $S_{2}$ (see Fig. 2):

$$
2 \pi n=\int_{\mathbf{S}^{2}} d A=\int_{S_{1}} d A+\int_{S_{2}} d a \rightarrow \int_{S_{1}} d A=-\int_{S_{2}} d A+2 \pi n
$$

so both equations for the phase:

$$
\gamma=\int_{C} A=\int_{S_{1}} d A \text { or } \quad-\int_{S_{2}} d A
$$

give the same result for $e^{i \gamma}$. In particular for the case of the 2-level degeneracy it's easy to show that the possible $\pi_{2}\left(\mathbf{C} \mathbf{P}^{N-1}\right)$ winding numbers are \pm 1 so that $\int_{\mathbf{S}^{2}}= \pm 2 \pi$.

These global obstructions can be viewed physically in terms of level crossings (two levels becoming degenerate somewhere on the parameter space). Two-level degeneracies occur as points generically in spaces of dimension 3, so that by surrounding the degeneracies by two-spheres we encounter the obstruction on $\mathbf{S}^{2}$ 's.

When the Hamiltonian is real (system symmetric under time reversal), the Hilbert space has a real structure and the non-degenerate wave-functions are defined up to a sign. In particular a 2-level real Hamiltonian defines a mapping

Fig. 2

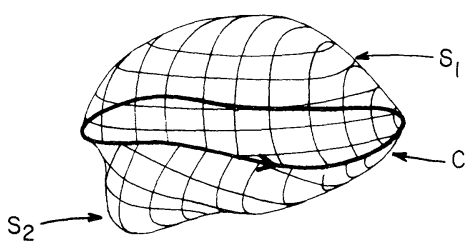


(under adiabatic transport), $f: M \rightarrow \mathbf{R P}^{1},\left(\mathbf{R P}^{1}\right.$ is a real projective space, the space of real 2-vectors of unit norm, up to a sign). The ability of globally defining the sign of the wave-function is equivalent to the ability of lifting $f$ to $\tilde{f}: M \rightarrow \mathbf{S}^{1}, \mathbf{S}^{1}$ being the space of real 2-vectors of unit norm.

From the exact sequence of the following bundle:

we learn that:

$$
\mathbf{Z}_{2} \equiv \mathbf{O}(1) \stackrel{i}{\rightarrow} \mathbf{S}^{1} \stackrel{p}{\rightarrow} \mathbf{R P}^{1}
$$

$$
\begin{aligned}
& \pi_{i}\left(\mathbf{R} \mathbf{P}^{1}\right) \approx \mathbf{0} \text { for } \quad i>1 \\
& 0 \rightarrow \pi_{1}\left(\mathbf{S}^{1}\right) \stackrel{p^{*}}{\rightarrow} \pi_{1}\left(\mathbf{R P}^{1}\right) \stackrel{\partial}{\rightarrow} \pi_{0}(\mathbf{O}(1)) \rightarrow 0,
\end{aligned}
$$

and since $\pi_{1}\left(\mathbf{S}^{1}\right) \approx \mathbf{Z}, \pi_{1}\left(\mathbf{R} \mathbf{P}^{1}\right) \approx \mathbf{Z}, \pi_{0}(\mathbf{O}(1)) \approx \mathbf{Z}_{2}$ we find that $\operatorname{Im} p^{*} \approx 2 \mathbf{Z}$.

So a non-trivial phase exists only on $\mathbf{S}^{1}$, generated by maps that have an odd winding number in $\pi_{1}\left(\mathbf{R P}^{1}\right)$. In this case the phase is a $\mathbf{Z}_{2}$ phase (a \pm sign), and the gauge connection a $\mathbf{Z}_{2}$ connection.

\section{The Non-Abelian Case}

As it was mentioned in a previous section, when a state is $n$-fold degenerate over the entire parameter manifold, then adiabatic transport may cause in general a $\mathbf{U}(n)$ rotation among the $n$ degenerate levels.

In this case we have $n$ degenerate states $|i\rangle_{\lambda}, \lambda$ specifying the point in the parameter space $M$. Defining the $U(n)$ connection 1-form:

$$
A_{i j} \equiv\left\langle j\left|\frac{\partial}{\partial t}\right| i\right\rangle d t=\left\langle j\left|d_{\lambda}\right| i\right\rangle,
$$

it's easy to show that after tracing a loop $C$ in $M$, the $n$ degenerate states will be rotated by a $U(n)$ matrix given by the Wilson loop, [3]:

$$
U_{C}=\mathbf{P} \exp \left[\int_{C} A\right] \text {. }
$$

Suppose that the Hamiltonian is an $N \times N$ Hermitian matrix. At each point of the parameter space we can always make the wave-functions of the $n$ degenerate states orthonormal. The question is : is it possible to have a smooth global system of orthonormal eigenstates over the entire parameter manifold?

The Hamiltonian in this case, (in analogy with the abelian case in Sect. 3), defines a mapping, from the parameter manifold into the Grassmann manifold $\mathbf{G}(N, n) \equiv \frac{\mathbf{U}(N)}{\mathbf{U}(N-n) \mathbf{U}(n)}$ which is the set of $n$-planes in $\mathbf{C}^{N}$. Then ability to globally define the orthonormal systems is equivalent to the ability of lifting the map $f: M \rightarrow \mathbf{G}(N, n)$ to $\tilde{f}: M \rightarrow \mathbf{V}(N, n) \equiv \frac{\mathbf{U}(N)}{\mathbf{U}(N-n)}$, the set of $n$-frames in $\mathbf{C}^{N}$. Intuitively it can be understood as follows. Starting from a fixed $n$-frame we can generate any other possible $n$-frame by $\mathbf{U}(N)$ rotations of the initial frame. But rotations in the orthogonal complement do not change the $n$-frame, so we must divide $\mathbf{U}(N)$ by $\mathbf{U}(N-n)$. The Hamiltonian also does not distinguish between two $n$-frames 
differing by a $\mathbf{U}(n)$ rotation so that the Hamiltonian really defines a mapping in $\frac{\mathbf{U}(N)}{\mathbf{U}(N-n) \mathbf{U}(n)}$. The abelian case discussed in the previous section is a special case of the above for $n=1$. Then $\frac{\mathbf{U}(N)}{\mathbf{U}(N-n)}=\mathbf{S}^{2 N+1}, \frac{\mathbf{U}(N)}{\mathbf{U}(N-1) \mathbf{U}(1)} \equiv \mathbf{C P}^{N-1}$.

We have the following principal fibration in this case:

$$
\mathbf{U}(n) \stackrel{i}{\rightarrow} \mathbf{V}(N, n) \stackrel{p}{\rightarrow} \mathbf{G}(N, n)
$$

and the theorem used in Sect. 3 applies here, giving the conditions for the ability to lift $f$ to $\tilde{f}$.

Again the obstruction can be viewed as due to level crossings. These introduce non-trivial topology in the parameter space and twist the Hilbert bundles.

For example, consider the transport of an $n$-fold degenerate level of a complex Hamiltonian and suppose that it becomes degenerate with another non-degenerate state at some point in the parameter space. The relevant part in the Hamiltonian near the degeneracy is the $(n+1) \times(n+1)$ sub-matrix describing the crossing levels. This sub-Hamiltonian is generically parametrized by $2 n$ parameters lying in $\frac{U(n+1)}{U(n) \mathbf{U}(1)} \equiv C P^{n}$. This means that such crossings occur generically at points in a $(2 n+1)$-dimensional space. So we have to look what happens on parameter manifolds being $\mathbf{S}^{m}, 1 \leqq m \leqq 2 n$, which encircle the degeneracy points. As it's shown in Appendix $C$, the only obstruction arises on 2-spheres in this case.

For an $n$-fold degenerate state, $(n>2)$, crossing a 2 -fold degenerate state we have to look on spheres $\mathbf{S}^{m}, 1 \leqq m \leqq 4 n$. It is also shown in Appendix $C$ that non-trivial holonomy arises on 2-spheres but also on 4-spheres (and possibly also on higher spheres).

If the Hamiltonian is real then the Hilbert space has a real structure, so that we have to consider the real Stieffel and Grassmann manifolds instead:

$$
\begin{gathered}
\mathbf{O}(n) \stackrel{i}{\rightarrow} \mathbf{R V}(N, n) \stackrel{p}{\rightarrow} \mathbf{R G}(N, n) \\
\mathbf{R V}(N, n) \equiv \frac{\mathbf{O}(N)}{\mathbf{O}(N-n)} \quad \mathbf{R G}(N, n) \equiv \frac{\mathbf{O}(N)}{\mathbf{O}(N-n) \mathbf{O}(n)}
\end{gathered}
$$

The connection in this case is an $\mathbf{O}(n)$ connection.

As an illustration, for the real case, consider the example given in [3], where an $n$-fold degenerate state of a real Hamiltonian is becoming degenerate at some point of the parameter space with another non-degenerate level. The relevant $(n+1) \times(n+1)$ part of the Hamiltonian depends in general on $n$ parameters lying in $\frac{O(n+1)}{O(n) O(1)} \equiv R P^{n}$. We have to look then on $M=\mathbf{S}^{m}, 1 \leqq m \leqq n$. As we show in Appendix $C$ a non-trivial phase ${ }^{2}$ appears only for $m=n$ and for $n \neq 7(\bmod 8)$. For $n=7(\bmod 8)$ no phase appears.

For a detailed discussion of some interesting special cases see Appendix C.

${ }^{2}$ By "phase" here, a $U(n)$ rotation of the degenerate subspace is meant 


\section{The Relation to Cohomology}

Our discussion of the representative paradigm of a non-trivial adiabatic phase in Sect. 2 hints the possible connection to cohomology.

We have a connection 1 -form, which has a string singularity somewhere on the sphere and which we cannot remove by a gauge transformation, but we can just move it around the sphere. The curvature 2-form is well-defined globally. Integrated over the $\mathbf{S}^{2}$ gives an integer which is the winding number of $\pi_{2}\left(\mathbf{C P}^{N-1}\right)$ :

$$
\frac{1}{2 \pi} \int_{\mathbf{s}^{2}} F=n
$$

We would expect these features to persist in the non-abelian case, too.

Our setting of the existence of a non-trivial adiabatic phase in terms of the impossibility of liftings of maps, gives the hint for an answer. This can be achieved using Obstruction Theory which is formulated in terms of Cohomology.

We will state the mathematical result, pertinent in our case:

Consider the following fibration and a map $f$ from a manifold $M$ to the base space of the bundle.

$$
M \stackrel{f}{\rightarrow} \mathbf{B} \stackrel{p}{\leftarrow} \mathbf{A} \stackrel{i}{\leftarrow} \mathbf{F} .
$$

We will suppose that $M$ is an $\mathbf{S}^{m}$.

The obstruction to lift the map $f$ to $\widetilde{f}: M \rightarrow \mathbf{A}$ is represented by the non-vanishing of the obstruction cochain $c(f)$, which is an element of $H^{n+1}\left(M, \pi_{n}[\mathbf{F}]\right)$, (the coefficient bundle in our case is trivial). In the complex case, $n=1$ since $\pi_{1}[U(N)]=\mathbf{Z}$ whereas in the real case, $n=0$ since $\pi_{0}[O(N)]=\mathbf{Z}_{2}$.

Using the results of Appendix A on obstruction theory we can conclude that in the complex case, the primary obstruction is in general non-vanishing only on $S^{2}$ corresponding to the first Chern class of the complex Hilbert bundle over $S^{2}$ while in the real case the primary obstruction is generically non-vanishing on $S^{1}$ corresponding to the first Stieffel-Whitney class of the real Hilbert bundle over $S^{1}$.

The forms that can represent the obstruction cochain $c(f)$ in the non-abelian case are the pullbacks under $f^{*}$ of the corresponding cohomology generators of the Grassmann manifold, which integrated over $\mathbf{S}^{m}$ will give integers (when they can be represented by differential forms).

To understand better the higher obstructions, let's consider the case where the primary obstruction vanishes, $\operatorname{Tr}(F)=0$. In this case our $U(n)$ bundle is equivalent to an $S U(n)$ bundle. Since $S U(n)$ is 3-contractable the obstruction in this case is represented by the second Chern class, $\operatorname{Tr}\left(F^{2}\right)$, which is a 4-form and arises on $S^{4}$. The generalization to higher obstructions is thus obvious. When the cohomology coefficients are torsion no interpretation using differential forms is available.

These non-trivial Chern classes, in the stable range, correspond to instanton-like configurations characteristic of non-trivial gauge bundles. Upon integrating them over $M$ we get integers which classify the respective $U(n)$ bundles.

The same reasoning applies to the real case. The corresponding obstruction cochains are the Stieffel-Whitney classes of the real Hilbert bundle.

It is just a routine check in order to verify that the cohomological picture gives the same results as the homotopy picture developed in Sects. 3 and 4. The advantage 
of the cohomology approach is that it displays explicitly the role of the curvature 2-form, $F$, the homotopy picture being easier to do computations with.

To conclude, whenever the obstruction cochain belongs to a cohomology group with integer coefficients, we can represent it with a differential form built out from the curvature form, $F$, corresponding to instanton-like configurations and which integrated over the parameter manifold will give integers related to the winding numbers of the map it obstructs.

When the obstruction is torsion, more clever techniques are needed to trace it. As was pointed out to me by L. Alvarez-Gaumé, the possible answer is using "generalized" $\eta$-invariants, capable of "seeing" torsion. Prototypes of such techniques where used by E. Witten in tracing the $S U(2)$ anomaly, [17] and global gravitational anomalies in ten dimensions, [18].

\section{Another Look at Anomalies}

As discussed already by $[6,7]$, the quantum adiabatic phase gives an elegant way of interpreting anomalies in the Hamiltonian picture.

The situation can be set as follows: Consider a chiral gauge theory with threedimensional space compactified to a sphere (or a product of spheres). The spectrum is discrete and we are working in the temporal gauge. Our aim is to construct a physical Hilbert space that is gauge invariant. The residual gauge transformations that respect the temporal gauge are time independent gauge transformations which are mappings from space $\left(\mathbf{S}^{3}\right)$ to the gauge group $G$ of the theory. We will consider $\mathbf{G}^{3} \equiv\left\{\right.$ maps : $\left.\mathbf{S}^{3} \rightarrow G\right\}$ and $\mathbf{A}^{3}$, (the space of static gauge potentials in the $A^{0}=0$ gauge), as our parameter space.

By adiabatically transporting around the parameter space $\mathbf{G}^{3}$ the vacuum state of the theory $|0\rangle$ we'd better not acquire a phase because that will indicate loss of gauge invariance. Otherwise stated the phase of the vacuum state should be globally defined over $\mathbf{G}^{3}$.

Let's suppose that the vacuum state is non-degenerate over $\mathbf{A}^{\mathbf{3}}$ (with the possible exception of some submanifold), and is an element of $\mathbf{C P}{ }^{\infty}$. From the analysis of Sect. 3 we know that in this case a non-trivial adiabatic phase arises due to the impossibility of global definition of phase over only the $\mathbf{S}^{2}$. There are noncontractable 2-spheres in $\mathbf{G}^{\mathbf{3}}$, if $\pi_{2}\left[\mathbf{G}^{3}\right]$ is non-trivial (if a sphere is contractable then it obviously creates no obstruction). Since $\pi_{2}\left[\mathbf{G}^{3}\right]=\pi_{5}[G]$ under suitable boundary conditions for the gauge transformations, theories with $\pi_{5}(G)$ being non-trivial have a potential danger of having anomalies. To establish their existence though we have to show that either the map induced by the Hamiltonian of the theory belongs to a non-trivial class of $\pi_{2}\left(\mathbf{C} \mathbf{P}^{\infty}\right)$ or to show that there is an accidental degeneracy somewhere that triggers a non-trivial adiabatic phase and thus a loss of gauge invariance in the theory. The existence of such a degeneracy in chiral gauge theories was established through an index theorem by [15].

If the vacuum state turns out to be gauge invariant, then the full Hilbert space can be constructed to be gauge invariant [6].

The $U(1)$ anomaly can be viewed in the same spirit, by looking for noncontractable tori in $\mathbf{G}^{3}$. We already know that $\left[\mathbf{S}^{1} \times \mathbf{S}^{1}, \mathbf{C} \mathbf{P}^{\infty}\right] \approx \mathbf{Z}$ and under 
suitable boundary conditions $\left[\mathbf{S}^{1} \times \mathbf{S}^{1}, \mathbf{G}^{3}\right] \approx\left[\mathbf{S}^{1} \times \mathbf{S}^{4}, \mathbf{U}(1)\right] \approx \mathbf{Z}$. The non-triviality of the later homotopy group shows that there are non-contractable tori in $\mathbf{G}^{\mathbf{3}}$ while the non-triviality of the former shows that an adiabatic phase is possible. The existence of a degeneracy that makes the phase to appear in a chiral $U(1)$ theory was shown by [16].

In the case of the non-perturbative $S U(2)$ anomaly the Hilbert space has a real structure and the vacuum state is an element of $\mathbf{R} \mathbf{P}^{\infty}$ which is again an EilebergMc Lane space: $\mathbf{R} \mathbf{P}^{\infty}=\mathbf{K}\left(\mathbf{Z}_{2}, 1\right)$, so that the only case we have to worry about is that of $\mathbf{S}^{1}$. Since $\pi_{1}\left(\mathbf{G}^{3}\right) \approx \pi_{4}(G)$ if $\pi_{4}(G)$ is non-trivial then there exist non-contractable $\mathbf{S}^{1}$ 's in $\mathbf{G}^{3}$, and thus there is a possibility of a non-trivial adiabatic phase to exist.

That this is true was shown by [17] by establishing the degeneracy giving rise to the non-trivial adiabatic phase in this case. Now the phase is a $\mathbf{Z}_{2}$ phase, it cannot be represented by differential forms, and it is "non-perturbative," because there are no infinitesimal closed paths on $\mathbf{S}^{\mathbf{1}}$.

A comment is in order here. We supposed that the vacuum state is not degenerate over $\mathbf{A}^{3}$. If the vacuum state is actually degenerate, then from our previous analysis we can conclude that there may be obstructions on higher spheres, in defining a gauge invariant Hilbert space. To assert that such cases are realizable requires some further analysis which we will carry out in a subsequent publication.

\section{The N-N Theorem and the Quantum Adiabatic Phase}

In this section we use the notion of the adiabatic phase and the underlying gauge structure to give an alternative easy proof of the N-N theorem, [12].

The theorem states that a lattice theory of chiral fermions in $3+1$ dimensions is impossible (due to species doubling), under the following assumptions:

(i) Locality of the Hamiltonian.

(ii) Translation invariance.

(iii) Hermiticity of the Hamiltonian.

(iv) Exact conservation of chiral charges.

(v) The fermion fields are complex.

(vi) The charges are bilinear in the fermion fields, locally defined an quantized.

Locality ensures the continuity of the fermion wave-functions in the Brillouin zone. Translation invariance makes the surface of the Brillouin zone a 2-torus, which is essential in the proof as can be seen below. Requirement (iv) means that energy-momentum eigenstates are also charge eigenstates. This along with local definition and quantization of charges are important for the identification of 2-level degeneracies in the Brillouin zone as RH and LH Weyl excitations in the continuum limit. The fermion wave-function being complex implies that the gauge field induced by adiabatic transport is non-zero. (Remember that in the real case the adiabatic phase on $\mathbf{S}^{1}$ is torsion and the connection form vanishes.)

The strategy of proving the claim above is, going to momentum space (Brillouin zone) and counting topologically the number of $\mathrm{LH}$ and $\mathrm{RH}$ fermion species which are associated with two-level degeneracies.

To see this, let's analyze the situation near a two-level degeneracy point, $\vec{p}=\vec{p}_{\mathrm{deg}}$, (these are the only generic degeneracies that occur in a 3-dimensional space). Near 
$\vec{p}_{\text {deg }}$, we can restrict to the relevant $2 \times 2$ part of the Hamiltonian.

$$
\begin{aligned}
& H^{(2)}(\vec{p}) u^{+}(\vec{p})=\omega_{+}(\vec{p}) u^{+}(\vec{p}), \\
& H^{(2)}(\vec{p}) u^{-}(\vec{p})=\omega_{-}(\vec{p}) u^{-}(\vec{p}),
\end{aligned}
$$

where $u^{ \pm}(\vec{p})$ are the fermion wave-functions corresponding to the upper $(+)$ or lower (-) level. Expanding to first order in $\vec{p}-\vec{p}_{\text {deg }}$ :

$$
H^{(2)}(\vec{p})=\omega_{\mathrm{deg}}\left(\vec{p}_{\mathrm{deg}}\right)+\left(\vec{p}-\vec{p}_{\mathrm{deg}}\right)_{\kappa} \sigma^{\alpha} C_{\alpha}^{\kappa}+\left(\vec{p}-\vec{p}_{\mathrm{deg}}\right) \cdot \vec{\alpha} \times{ }^{D}\left[\left(\vec{p}-\vec{p}_{\mathrm{deg}}\right)\right],
$$

so that $(7.1 \mathrm{a}, \mathrm{b})$ becomes:

$$
\left(\vec{p}-\vec{p}_{\mathrm{deg}}\right)_{\kappa} \sigma^{\alpha} C_{\alpha}^{\kappa} u^{ \pm}(\vec{p})=\left[\omega_{ \pm}(\vec{p})-\omega_{\mathrm{deg}}\left(\vec{p}_{\mathrm{deg}}\right)-\left(\vec{p}-\vec{p}_{\mathrm{deg}}\right) \cdot \vec{\alpha}\right] u^{ \pm}(\vec{p})
$$

Redefining:

$$
\begin{gathered}
\omega(\vec{p})=\omega(\vec{p})-\omega_{\mathrm{deg}}\left(\vec{p}_{\mathrm{deg}}\right), \\
\vec{p}=\vec{p}-\vec{p}_{\mathrm{deg}},
\end{gathered}
$$

and introducing a new coordinate system:

$$
\begin{gathered}
P_{0}=\omega-\vec{\alpha} \cdot \vec{p}, \\
P_{\alpha}=p_{\kappa} C_{\alpha}^{\kappa},
\end{gathered}
$$

(7.3) becomes the Weyl equation:

$$
\vec{\sigma} \cdot \vec{P} u(\vec{p})=P_{0} u(\vec{p})
$$

It's easy to see that if $\operatorname{det} C>0(<0)$ the $\vec{P}$ coordinate system is RH(LH). So that for $P_{0}>0,(+$ level), $\vec{\sigma} \cdot \vec{p}>0$ and if the $\vec{P}$ system is $\mathrm{RH}$, then $\vec{\sigma} \cdot \vec{P}>0$ (positive helicity), otherwise $\vec{\sigma} \cdot \vec{P}<0$ (negative helicity).

In conclusion:

$\operatorname{det} C>0$ the degeneracy represents a LH fermion species, $(+$ level $)$ and its anti-particle $(-$ level $)$,

$\operatorname{det} C<0$ the degeneracy represents a $R H$ fermion species, $(+$ level $)$ and its anti-particle (-level) .

At each two-level degeneracy (according to Sect. 2), we have a monopole "magnetic field" which upon integration gives the adiabatic phase:

$$
\begin{gathered}
\phi^{+}=-\frac{1}{2} \operatorname{sign}(\operatorname{det} C) \Omega \equiv \int_{D} d \vec{S} \cdot \vec{B}^{+}, \\
\phi^{-}=\frac{1}{2} \operatorname{sign}(\operatorname{det} C) \Omega \equiv \int_{D} d \vec{S} \cdot \vec{B}^{-},
\end{gathered}
$$

where $\partial D=\mathbf{C}$ is the closed contour around which we adiabatically transport, + and - refer to the upper and lower level, and $\Omega$ is the solid angle subtend by $\mathbf{C}$ as seen from the degeneracy. 
If we now surround the point degeneracies in the Brillouin zone by small 2-spheres we have "magnetic flux" coming out:

$$
\begin{gathered}
\Phi^{+}=-\frac{1}{2} \operatorname{sign}(\operatorname{det} C) 4 \pi \equiv \int_{\mathbf{S}^{2}} d \vec{S} \cdot \vec{B}^{+}, \\
\Phi^{-}=\frac{1}{2} \operatorname{sign}(\operatorname{det} C) 4 \pi \equiv \int_{\mathbf{S}^{2}} d \vec{S} \cdot \vec{B}^{-} .
\end{gathered}
$$

Let's now concentrate on these fluxes. Consider the "magnetic field" generated by adiabatic transport of the $n^{\text {th }}$ level. It is a sum of monopole fields over the various degeneracies that the $n^{\text {th }}$ level participates in, as it moves around adiabatically. As an example, for the situation depicted in Fig. 4 the magnetic field is:

$$
\vec{B}_{n}=\vec{B}_{1}^{+}+\vec{B}_{2}^{+}+\vec{B}_{3}^{-}+\vec{B}_{4}^{-},
$$

where the lower index on the right-hand side labels the degeneracies. Then, applying Gauss' theorem:

$$
\int_{S_{B}} d \vec{S} \cdot \vec{B}_{n}-\sum_{i} \int_{\mathbf{S}_{i}^{2}} d \vec{S} \cdot \vec{B}_{n}=\int_{V} d V \vec{\nabla} \cdot \vec{B}_{n}=0
$$

where, $S_{B}$ is the surface of the Brillouin zone, $\mathbf{S}_{i}^{2}$ is the 2-sphere surrounding the $i^{\text {th }}$ degeneracy, the sum is over all degeneracies that the $n^{\text {th }}$ level meets in its way around, and $V$ is the volume of the Brillouin zone minus the volumes of the small

Fig. 3

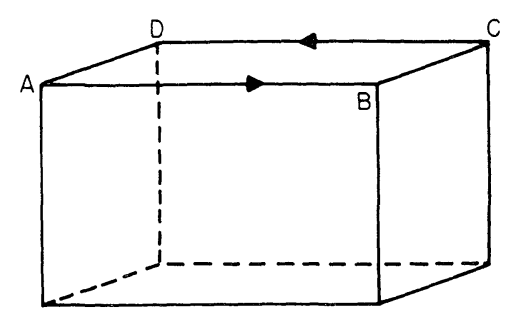

Fig. 4

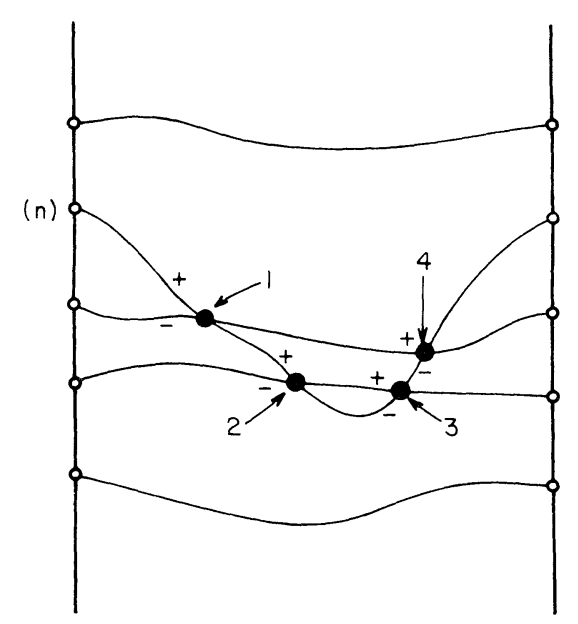


spheres surrounding the respective degeneracies. The last volume integral is zero because the $\vec{B}_{n}$ field is divergenceless outside the small 2-spheres, (corresponding to $d F=0)$.

Denote sign $(\operatorname{det} C)$ at the $i^{\text {th }}$ degeneracy by $\sigma_{i}$. From $(7.10)$ and $(7.8 \mathrm{a}, \mathrm{b})$ we have:

$$
\sum_{i} 2 \pi \sigma_{i}^{+}-\sum_{j} 2 \pi \sigma_{j}^{-}=\int_{S_{B}} d \vec{S} \cdot \vec{B}_{n}=\sum_{\text {faces }} \int d \vec{S} \cdot \vec{B}_{n}=\sum_{\text {faces }} \int_{C_{j}} d \vec{l} \cdot \vec{A}_{n},
$$

where we sum over the rectangular faces of the Brillouin zone, $C_{j}$ is the contour around the $j^{\text {th }}$ face, $\vec{A}_{n}$ is the respective gauge potential and the sums in the left-hand side are over degeneracies where the $n^{\text {th }}$ level meets the $(n-1)^{\text {th }},(+)$, or the $(n+1)^{\text {th }}$ level, (-) respectively. Now consider, for example, the integral $\int_{A B C D} d \vec{l} \cdot \vec{A}_{n}$ (see Fig. 3). This is zero because due to the periodicity of the Brillouin zone it's like retracing back and forth the two principal circles of a 2 -torus. This is true obviously for every face so:

$$
\sum_{i} \sigma_{i}^{+}-\sum_{j} \sigma_{j}^{-}=0
$$

which states that (using the aforementioned identification of $\mathrm{LH}$ and $\mathrm{RH}$ fermion species, that is, $\sigma_{i}>0 \rightarrow L H$ species, $\sigma_{i}<0 \rightarrow R H$ species):

$$
N_{L}(n, n+1)-N_{R}(n, n+1)=N_{L}(n, n-1)-N_{R}(n, n-1),
$$

where $N_{L, R}(n, n+1)$ is the number of $\mathrm{LH},(\mathrm{RH})$, degeneracies between the $n^{\text {th }}$ and the $(n+1)^{\text {th }}$ level. From this then and the fact that $N_{L}(1,0)=N_{R}(1,0)=0$ trivially, (because the spectrum begins from the first level) we obtain:

$$
N_{L}(n, n+1)=N_{R}(n, n+1)
$$

by induction. This is the final result of the N-N theorem implying equal numbers of LH and RH fermion species in a lattice theory.

\section{Conclusions}

We have taken advantage of the topological nature of the quantum adiabatic phase to state a rigorous theorem on its appearance over compact parameter manifolds with non-trivial topology. The existence of the phase is associated to the impossibility of global definition of phase of the adiabatically transported wavefunction or equivalently to the impossibility of lifting the mapping induced by adiabatic transport from the space of normalized wave-vectors, $\left(\mathbf{C P}^{N}\right.$ or $\left.\mathbf{G}(N, n)\right)$ to $\mathbf{C}^{N}$ or $\mathbf{V}(N, n)$ respectively.

The question of topologically classifying the adiabatic phase reduces to classifying the gauge bundles on spheres which are the pullback of the respective principal bundles over Grassmann manifolds.

Level crossings during adiabatic transport are responsible for this rich topological structure. Their presence twists the Hilbert bundles over the parameter space obstructing in this way the global definition of the phase of the wave function (or the internal orientation of the $n$-degenerate levels). 
Using obstruction theory we can link the existence of a non-trivial Berry phase to the non-trivial cohomology of the parameter manifold generated by the gauge connection of the Hilbert bundle. In particular, in the non-abelian case we have instanton-like configurations, induced by level crossings, paralleling the appearance of monopole bundles in the 2-level case [1,2]. The whole set-up reduces to the results of $[6,7]$ when applied to gauge theories with perturbative and nonperturbative anomalies.

\section{Appendix A}

In this appendix we give some pertinent mathematical results useful for Sect. 3-5. More details can be found in $[10,11]$.

$1^{\text {st }}$ Covering Homotopy Theorem. Consider two fibre bundles $B$ and $B^{\prime}$ having the same fiber and group. Let the base space $X$ of $B$ be $a C_{\sigma}$ space (normal, locally compact and such that any covering of $X$ by open sets is reducible to a countable covering). Let $h_{0}: B \rightarrow B^{\prime}$ be a bundle map and $\bar{h}: X \times I \rightarrow X^{\prime}$ be a homotopy of the induced map $\bar{h}_{0}: X \rightarrow X^{\prime}$. Then there exists a homotopy $h: B \times I \rightarrow B^{\prime}$ whose induced homotopy is $\bar{h}$ and $h$ is stationary with $\bar{h}$.

$2^{\text {nd }}$ Covering Homotopy Theorem. Let $B^{\prime}$ be a bundle over $X^{\prime}$, let $X$ be a $C_{\sigma}$ space and $f_{0}: X \rightarrow B^{\prime}$ a map. $\bar{f}: X \times I \rightarrow X^{\prime}$ a homotopy of $\bar{f}_{0}=p^{\prime} \circ f_{0}$. Then there exists a homotopy $f: X \times I \rightarrow B^{\prime}$ covering $\bar{f}$ (i.e. $\bar{f}=p^{\prime} \circ f$ ) and $f$ is stationary with $\bar{f}$.

The Exact Homotopy Sequence of a Principal fibration. Let $\mathbf{B} \equiv(X, B, F, p)$ be a principal fibre bundle with base space $X$ bundle space $B$ fibre and group $F$ and projection $p$.

$$
F \stackrel{i}{\rightarrow} B \stackrel{p}{\rightarrow} X
$$

Then the following sequence is exact:

$$
\ldots \stackrel{\partial}{\rightarrow} \pi_{i}(F) \stackrel{i^{*}}{\rightarrow} \pi_{i}(B) \stackrel{p^{*}}{\rightarrow} \pi_{i}(X) \stackrel{\partial}{\rightarrow} \pi_{i-1}(F) \rightarrow \ldots
$$

terminating at:

$$
\ldots \stackrel{p^{*}}{\rightarrow} \pi_{1}(X) \stackrel{\partial}{\rightarrow} \pi_{0}(F) \stackrel{i^{*}}{\rightarrow} \pi_{0}(B) .
$$

Below we give some results on obstruction theory in fibre spaces [11].

Let $F \stackrel{i}{\rightarrow} B \stackrel{p}{\rightarrow} X$ be a fibration, $\mathrm{K}$ a CW complex, and a map $f: K \rightarrow X$. Suppose we were able to lift the map $f$ to $\widetilde{f}_{n-1}: K_{n-1} \rightarrow B$, where $K_{n-1}$ is the $(n-1)^{\text {th }}$ skeleton of $K$. The obstruction of lifting the map to $\tilde{f}_{n}: K_{n} \rightarrow B$ (that is extending the map to the next $n$-cell) is represented by the obstruction cochain $\bar{c}^{n}(f) \in \Gamma^{n}\left[K, f^{*} \pi_{n-1}(\mathbf{F})\right]$. We have assumed that the fibre is $(n-1)$-simple so that $\pi_{n-1}(\mathbf{F})$ is a system of local coefficients in $B$ and $f^{*} \pi_{n-1}(\mathbf{F})$ is a system of local coefficients in $K$.

The main theorem is as follows: Consider a fibration, $F \stackrel{i}{\rightarrow} A \stackrel{p}{\rightarrow} B$, and a map $f: M \rightarrow B$. Let the fiber be $(n-1)$-connected (that is, the first non-trivial homotopy group is $\left.\pi_{n}[F]\right)$. If $H^{q+1}\left(M, \pi_{q}[F]\right) \approx 0$ for $n+1 \leqq q<\operatorname{dim} M$, then the map $f$ can be lifted to a map $\tilde{f}: M \rightarrow A, p \circ \tilde{f}=f$, if and only if the primary obstruction cochain 
vanishes:

$$
H^{n+1}\left(M, \pi_{n}[F]\right) \quad \bar{c}^{n+1}(f)=0 .
$$

Higher obstruction cochains become meaningful only when the primary obstruction vanishes.

\section{Appendix B}

In this appendix the theorem used in Sect. 3 is proven.

Consider a general principal fiber bundle $B \equiv(\mathbf{B}, \mathbf{A}, \mathbf{F})$ where $\mathbf{B}$ is the base space, $\mathbf{A}$ is the bundle space, $\mathbf{F}$ is the fibre and a the same time the structure group of the bundle and a manifold $M$ along with a mapping $f: M \rightarrow \mathbf{B}$.

$$
M \stackrel{f}{\rightarrow} \mathbf{B} \stackrel{p}{\leftarrow} \mathbf{A} \stackrel{i}{\leftarrow} \mathbf{F} .
$$

We will try to find the condition for the existence of the lifting $\tilde{f}$ of $f,(p \circ \tilde{f}=f)$ $\tilde{f}: M \rightarrow \mathbf{A}$. The projection $p$ induces a mapping $p^{*}:[M, \mathbf{A}] \rightarrow[M, \mathbf{B}]([M, \mathbf{B}]$ is the set of homotopy classes of mappings from $M$ to $\mathbf{B}$. In all of our applications it will have the structure of an abelian group).

Theorem. $f$ can be lifted if and only if it belongs to a class in $\operatorname{Im} p^{*}$.

Proof. Suppose that $f$ belongs to a class $[f] \in \operatorname{Im} p^{*}$. Then there is a map $g \in[f]$ which can be lifted to $\tilde{g}$, (if there is not such a map then $[f]$ does not belong to $\left.\operatorname{Im} p^{*}\right)$. Under $p^{*}[\tilde{g}]$ is mapped to $[f]$. By assumption we can connect $f$ and $g$ with a homotopy $h$ such that $h(0)=f, h(1)=g$. Then by the second homotopy covering theorem (see Appendix A) there is a homotopy $\tilde{h}$ such that $\tilde{h}(0)=\tilde{f}, \tilde{h}(1)=\tilde{g}$ and obviously $\tilde{f}$ is the lifting of $f$ since $p \circ \tilde{h}=h$.

Suppose now that $f$ does not belong to $\operatorname{Im} p^{*}$. It $f$ could be lifted to a map $\tilde{f}: M \rightarrow \mathbf{A}, p \circ \tilde{f}=f$, then $\widetilde{f}$ belongs to some class in $[M, \mathbf{A}]$ and there is a homotopy $\tilde{h}$ connecting $\tilde{f}$ to some other map $\tilde{g} \in[\tilde{f}]$. The projection of $\tilde{h}$ is a homotopy connecting $f$ to $g \equiv p \circ \tilde{g}$ which in turn means that $[f] \in \operatorname{Im} p^{*}$, false by assumption. Q.E.D.

Corollary 1. If $[M, \mathbf{A}] \approx 0$ and $[M, \mathbf{B}]$ is non-trivial then any map belonging to a nontrivial class in $[M, \mathbf{B}]$ cannot be lifted.

Corollary 2. If $[M, \mathbf{B}] \approx 0$ any map can be lifted.

Corollary 3. If $M=\mathbf{S}^{n}$ and $\pi_{n}(\mathbf{F}) \approx 0$ then $p^{*}$ is $1-1$.

Proof. From the exact homotopy sequence of a fibre bundle:

$$
\ldots \stackrel{\partial}{\rightarrow} \pi_{n}(\mathbf{F}) \stackrel{i^{*}}{\rightarrow} \pi_{n}(\mathbf{A}) \stackrel{p^{*}}{\rightarrow} \pi_{n}(\mathbf{B}) \stackrel{\partial}{\rightarrow} \pi_{n-1}(\mathbf{F}) \rightarrow \ldots
$$

we get: $\operatorname{Im} i^{*} \approx 0$ so $\operatorname{Ker} p^{*} \approx 0$.

Corollary 4. If $M=\mathbf{S}^{n}$ and $\pi_{n-1}(\mathbf{F}) \approx 0$ then $p^{*}$ is onto.

Proof. From the same exact sequence above, $\operatorname{Im} \partial \approx 0, \operatorname{Ker} \partial \approx \operatorname{Im} p^{*} \approx \pi_{n}(\mathbf{B})$, $p^{*}$ is onto. 


\section{Appendix C}

In this appendix we work some examples showing when a non-trivial adiabatic phase arises in certain cases.

Consider first the case of a real Hamiltonian, which is $(n+1) \times(n+1)$ dimensional, with $n$ degenerate over the parameter manifold. It describes the system near a degeneracy where the $n$ degenerate levels cross a single non-degenerate level. (This is the example discussed in [3].) As it was pointed out in Sect. 4 we have to look on parameter manifolds being spheres, $\mathbf{S}^{m}, 1 \leqq m \leqq n$. The relevant fibre bundle picture is:

$$
\mathbf{O}(n) \stackrel{i}{\rightarrow} \mathbf{R V}(n+1, n) \stackrel{p}{\rightarrow} \mathbf{R} \mathbf{G}(n+1, n),
$$

where $\mathbf{R V}(n+1, n) \equiv \frac{\mathbf{O}(n+1)}{\mathbf{O}(1)} \approx S O(n+1)$ and $\mathbf{R G}(n+1, n) \equiv \frac{\mathbf{O}(n+1)}{\mathbf{O}(n) \mathbf{O}(1)} \approx \mathbf{S}^{n} / \mathbf{Z}_{2}$ so that $\pi_{i}[\mathbf{R G}(n+1, n)] \approx \pi_{i}\left(\mathbf{S}^{n}\right)$ for $i \geqq 1$ and $\pi_{0}[\mathbf{R G}(n+1, n)] \approx \mathbf{Z}_{2}$.

On the sphere, $M=\mathbf{S}^{m}, m<n$ since $\pi_{m}[\mathbf{R G}(n+1, n)]$ is trivial, there is no phase, for every mapping $f: \mathbf{S}^{m} \rightarrow \mathbf{R G}(n+1, n)$. But for $M=\mathbf{S}^{n}$ the situation is different. The relevant exact homotopy sequence of the fibration is:

$$
\begin{aligned}
\ldots & \stackrel{\partial}{\rightarrow} \pi_{n}[S O(n)] \stackrel{i^{*}}{\rightarrow} \pi_{n}[S O(n+1)] \stackrel{p^{*}}{\rightarrow} \pi_{n}\left(\mathbf{S}^{n}\right) \stackrel{\partial}{\rightarrow} \pi_{n-1}[S O(n)] \\
& \stackrel{i^{*}}{\rightarrow} \pi_{n-1}[S O(n+1)] \rightarrow 0 .
\end{aligned}
$$

(i) For $n=0,4(\bmod 8), \pi_{n-1}[S O(n+1)] \approx \mathbf{Z}, \pi_{n-1}[S O(n)] \approx \mathbf{Z}+\mathbf{Z}$ and $(\mathrm{C} .2)$ becomes:

$$
\ldots \rightarrow \pi_{n}[S O(n+1)] \stackrel{p^{*}}{\rightarrow} \mathbf{Z} \rightarrow \mathbf{Z}+\mathbf{Z} \rightarrow \mathbf{Z} \rightarrow 0,
$$

which shows that $p^{*}$ is the zero map, so every map being homotopically non-trivial will give a non-trivial phase.

(ii) For $n=1,2(\bmod 8), \pi_{n-1}[S O(n+1)] \approx \mathbf{Z}_{2}$, and (B.2) becomes:

$$
\ldots \rightarrow \pi_{n}[S O(n+1)] \stackrel{p^{*}}{\rightarrow} \mathbf{Z} \stackrel{\partial}{\rightarrow} \pi_{n-1}[S O(n)] \stackrel{i^{*}}{\rightarrow} \mathbf{Z}_{2} \rightarrow 0 .
$$

There are three possibilities for $\pi_{n-1}[S O(n)]$ :

(a) $\pi_{n-1}[S O(n)] \approx \mathbf{Z}$. Then $\operatorname{Im} i^{*}=\mathbf{Z}_{2}, \operatorname{Ker} i^{*} \approx 2 \mathbf{Z} \approx \operatorname{Im} \partial$ so that $\operatorname{Ker} \partial \approx 0$ $\approx \operatorname{Im} p^{*}$. Every non-trivial map gives a phase.

(b) $\pi_{n-1}[S O(n)] \approx \mathbf{Z}+\mathbf{Z}_{2}$ then $\operatorname{Ker} i^{*} \approx \mathbf{Z}_{2}$ or $2 \mathbf{Z} \approx \operatorname{Im} \partial$ so that $\operatorname{Ker} \partial$ $\approx \operatorname{Im} p^{*} \approx 0$ and the situation is the same as in (a).

(c) $\pi_{n-1}[S O(n)] \approx \mathbf{Z}_{2}+\mathbf{Z}_{2}$ then $\operatorname{Ker} i^{*} \approx \mathbf{Z}_{2} \approx \operatorname{Im} \partial$, so that $\operatorname{Ker} \partial \approx \operatorname{Im} p^{*} \approx 2 \mathbf{Z}$, and every map with an odd winding number gives a non-trivial phase.

(iii) For $n=3,5,6(\bmod 8) \pi_{n-1}[S O(n+1)] \approx 0$ and from $(\mathrm{C} .2)$ :

$$
\ldots \rightarrow \pi_{n}[S O(n+1)] \stackrel{p^{*}}{\rightarrow} \mathbf{Z} \stackrel{\partial}{\rightarrow} \pi_{n-1}[S O(n)] \rightarrow 0,
$$

and since in this case $\pi_{n-1}[S O(n)]$ is always non-trivial there are mapping in this case too, that give a phase.

(iv) For $n=7(\bmod 8) \pi_{n-1}[S O(n)] \approx 0$ so there is no phase in this case.

For the case of a real Hamiltonian, where a doubly degenerate state crosses with another doubly degenerate state we can easily see using the procedure above, that the only non-trivial obstruction exists on $\mathbf{S}^{1}$. 
As a second example consider a complex Hamiltonian with $n$ degenerate levels, crossing at some point another non-degenerate level. As mentioned in Sect. 4, such crossings occur generically at isolated points in parameter spaces of dimension $2 n+1$. Consequently, we have to investigate parameter manifolds being spheres $\mathbf{S}^{m}, 1 \leqq m \leqq 2 n . \mathbf{V}(n+1, n) \equiv \frac{\mathbf{U}(n+1)}{\mathbf{U}(1)}$ with:

$$
\begin{aligned}
& \pi_{i}[\mathbf{V}(n+1, n)] \approx 0 \quad i=0,1,2, \\
& \pi_{i}[\mathbf{V}(n+1, n)] \approx \pi_{i}[\mathbf{U}(n+1)] \quad i \geqq 3,
\end{aligned}
$$

$\mathbf{G}(n+1, n) \equiv \frac{\mathbf{U}(n+1)}{\mathbf{U}(n) \mathbf{U}(1)} \equiv \mathbf{C P}^{n}$,

$$
\begin{aligned}
& \pi_{i}\left(\mathbf{C} \mathbf{P}^{n}\right) \approx \pi_{i}\left(\mathbf{S}^{2 n+1}\right) \quad i \geqq 3 \\
& \pi_{2}\left(\mathbf{C} \mathbf{P}^{n}\right) \approx \mathbf{Z}, \quad \pi_{1}\left(\mathbf{C} \mathbf{P}^{n}\right) \approx 0 .
\end{aligned}
$$

Since $\pi_{i}\left(\mathbf{S}^{2 n+1}\right) \approx 0$ for $3 \leqq i \leqq 2 n$, the only obstruction exists on 2 -spheres, where any non-trivial map in $\pi_{2}\left(\mathbf{C} \mathbf{P}^{n}\right)$ cannot be lifted.

In the case where an $n$-degenerate level crosses a doubly-degenerate level, the general $(n+2) \times(n+2)$ relevant part of the Hamiltonian is parametrized in $\frac{\mathbf{U}(n+2)}{\mathbf{U}(n) \mathbf{U}(2)}$ which has dimension $4 n$, so that such degeneracies occur generically at points in parameter spaces of dimension $4 n+1$. So we must look on spheres, $\mathbf{S}^{m}, 1 \leqq m \leqq 4 n$. Using the exact sequence of the bundle:

$$
\mathbf{U}(2) \stackrel{i}{\rightarrow} \mathbf{U}(n+2) \stackrel{p}{\rightarrow} \mathbf{V}(n+2, n),
$$

we can get the following information on homotopy groups:

$$
\pi_{i}[\mathbf{V}(n+2, n)] \approx 0 \text { for } 0 \leqq i \leqq 4
$$

We will distinguish two cases:

(i) $n=2$. Then, $\mathbf{V}(4,2) \equiv \frac{\mathbf{U}(4)}{\mathbf{U}(2)}$ and there is a special fibration in this case,

$$
\mathbf{S}^{5} \stackrel{i}{\rightarrow} \mathbf{V}(4,2) \stackrel{p}{\rightarrow} \mathbf{S}^{7},
$$

which has a global section. Using the information above we can read the relevant homotopy groups:

$$
\begin{array}{ll}
\pi_{5}[\mathbf{V}(4,2)] \approx \mathbf{Z}, & \pi_{6}[\mathbf{V}(4,2)] \approx \mathbf{Z}_{2}, \\
\pi_{7}[\mathbf{V}(4,2)] \approx \mathbf{Z}+\mathbf{Z}_{2}, & \pi_{8}[\mathbf{V}(4,2)] \approx \mathbf{Z}_{2}+\mathbf{Z}_{24} .
\end{array}
$$

Using now the fibration,

$$
\mathbf{U}(2) \stackrel{i}{\rightarrow} \mathbf{V}(4,2) \stackrel{p}{\rightarrow} \mathbf{G}(4,2),
$$

and the fact that (C.4) is a trivial bundle we can infer that $p^{*}: \pi_{n}[\mathbf{V}(4,2)]$ $\rightarrow \pi_{n}[\mathbf{G}(4,2)]$ is a map such that $\left.\pi_{n}[\mathbf{G} 4,2)\right] \approx \operatorname{Im} p^{*}+\pi_{n-1}\left[\mathbf{S}^{1} \times \mathbf{S}^{3}\right]$. Non-triviality of $\pi_{n-1}\left[\mathbf{S}^{1} \times \mathbf{S}^{3}\right]$ then signals the existence of a topologically non-trivial adiabatic phase on the $n$-sphere. Consequently we have no phase on $\mathbf{S}^{\mathbf{1}}, \mathbf{S}^{\mathbf{3}}$, a $\mathbf{Z}$ phase on $\mathbf{S}^{2}$ 
and $\mathbf{S}^{4}$, a $\mathbf{Z}_{2}$ phase on $\mathbf{S}^{5}, \mathbf{S}^{6}, \mathbf{S}^{8}$ and a $\mathbf{Z}_{12}$ phase on $\mathbf{S}^{7}$. The above list exhausts the possibilities of a non-trivial phase when a two-degenerate level crosses another 2-degenerate level.

(ii) $n>2$. We can read some relevant homotopy groups from (B.3),

$$
\begin{aligned}
& \pi_{5}[\mathbf{V}(n+2,2)] \approx \mathbf{Z}, \quad \pi_{6}[\mathbf{V}(n+2,2)] \approx \mathbf{Z}_{2}, \\
& \pi_{7}[\mathbf{V}(n+2,2)] \approx \mathbf{Z}+\mathbf{Z}_{2}, \pi_{8}[\mathbf{V}(n+2,2)] \approx \mathbf{Z}_{2} .
\end{aligned}
$$

Using the exact sequence of the bundle:

$$
\mathbf{U}(n) \stackrel{i}{\rightarrow} \mathbf{V}(n+2, n) \stackrel{p}{\rightarrow} \mathbf{G}(n+2, n),
$$

we can get some partial information:

For $n=3$, we have non-trivial phases on $\mathbf{S}^{2}, \mathbf{S}^{4}$ and trivial ones on $\mathbf{S}^{1}, \mathbf{S}^{3}, \mathbf{S}^{5}$.

For $n>3$, we have non-trivial phases on $\mathbf{S}^{2}, \mathbf{S}^{4}$ and trivial ones on $\mathbf{S}^{1}, \mathbf{S}^{3}, \mathbf{S}^{5}, \mathbf{S}^{6}$. Due to incomplete knowledge of higher homotopy groups we cannot say anything for phases on an $m$-sphere with $6<m<4 n$.

It is obvious now that in the general situation when an $n$-fold degenerate level crosses an $m$-fold degenerate level $(n \geqq m)$, we ought to look on parameter manifolds $M=\mathbf{S}^{r}$, where $1 \leqq r \leqq 2 m n$. Considerable information is obtained from some known homotopy groups:

$$
\begin{aligned}
& \pi_{i}[\mathbf{V}(m+n, n)] \approx 0 \text { for } i \leqq 2 m \text { and } \pi_{2 m+1}[\mathbf{V}(m+n, n)] \approx \mathbf{Z} \\
& \pi_{i}[\mathbf{G}(n+m, n)] \approx \pi_{i-1}[\mathbf{U}(n)] \text { for } i \leqq 2 m
\end{aligned}
$$

We can assert that non-trivial holonomy appears on $\mathbf{S}^{2 r}, 1 \leqq r \leqq m$. For $n>m$ no obstruction appears on $\mathbf{S}^{2 m+1}$. For $n=m, \pi_{2 n}[\mathbf{U}(n)] \approx \mathbf{Z}_{n !}$ and by looking at:

$$
\ldots \rightarrow \pi_{2 n+1}[\mathbf{U}(n)] \stackrel{i^{*}}{\rightarrow} \pi_{2 n+1}[\mathbf{V}(2 n, n)] \stackrel{p^{*}}{\rightarrow} \pi_{2 n+1}[\mathbf{G}(2 n, n)] \stackrel{\circ}{\rightarrow} \mathbf{Z}_{n !} \rightarrow 0
$$

we can see that any map which has a winding number not a multiple of $n$ ! gives a non-trivial phase in this case.

Proceeding in this way we can get information potentially for every case.

Let's consider as a final example the case of an infinite dimensional Hamiltonian $(N \rightarrow \infty$, with a countable number of eigenstates). In the real case we know that $\mathbf{R V}(\infty, n)$ is a contractable space so that all its homotopy groups are trivial. Since $\pi_{i}[\mathbf{R G}(\infty, n)] \approx \pi_{i-1}[\mathbf{O}(n)]$, whenever this homotopy group is non-trivial we get a phase for any homotopically non-trivial mapping.

In the complex case, $\mathbf{V}(\infty, n)$ is again contractable and $\pi_{i}[\mathbf{G}(\infty, n)]$ $\approx \pi_{i-1}[\mathbf{U}(n)]$, the situation is the same as in the real one.

In particular for $n=1$ there is a $\mathbf{Z}_{2}$ phase only on $\mathbf{S}^{1}$ in the real case and a $\mathbf{Z}$ phase only on $\mathbf{S}^{2}$ in the complex case.

Acknowledgements. I would like to thank A. Polychronakos and $\mathrm{H}$. Sonoda for a lot of illuminating discussions on the subject; J. Preskill for pointing out to me a lot of the expected features of the adiabatic phase and for constant encouragement, L. Alvarez-Gaumé for explaining to me how one could see phases that are torsion, and M. Anderson for patiently explaining parts of the mathematical machinery used. 


\section{References}

1. Berry, M.: Proc. R. Soc. Lond. A392, 45 (1984)

2. Simon, B.: Holonomy, the quantum adiabatic theorem, and Berry's phase. Phys. Rev. Lett. 51, 2167 (1983)

3. Wilczek, F., Zee, A.: Appearance of gauge structure in simple dynamical systems. Phys. Rev. Lett. 52, 2111 (1984)

4. Moody, J., Shapere, A., Wilczek, F.: ITP preprint NSF-ITP-85-78

5. Kuratsuji, H., Iida, S.: Kyoto, Univ. preprint, KUNS-770

6. Nelson, P., Alvarez-Gaumé L.: Hamiltonian interpretation of anomalies. Commun. Math. Phys. 99, 103 (1985)

7. Sonoda, H.: The Wess-Zumino term and the Hamiltonian formulation for anomalies. Phys. Lett. 156B, 220 (1985)

8. Sonoda H.: Berry's phase in chiral gauge theories. Nucl. Phys. B266, 410 (1986)

9. Niemi, A.J., Semenoff, G.W.: Quantum holonomy and the chiral gauge anomaly. Phys. Rev. Lett. 55, 927 (1985)

10. Steenrod, N.: The topology of fibre bundles. Princeton, NJ : Princeton University Press 1951

11. Whitehead, G. : Elements of homotopy theory. Berlin, Heidelberg, New York: Springer 1978

12. Nielsen, H.B., Ninomiya, M.: Absence of neutrinos on a lattice (I). Proof by homotopy theory. Nucl. Phys. B185, 20 (1981)

13. Thouless, D.J., Kohmoto, M., Nightingale, M.P., den Nijs, M.: Quantized Hall conductance in a two-dimensional periodic potential. Phys. Rev. Lett. 49, 405 (1982)

14. Avron, J.E., Seiler, R., Simon, B.: Homotopy and quantization in condensed matter physics. Phys. Rev. Lett. 51, 51 (1983)

15. Alvarez-Gaumé, L., Ginsparg, P.: The topological meaning of non-abelian anomalies. Nucl. Phys. B243, 449 (1984)

16. Feng, Jn, Preskill, J.: Caltech preprint CALT-68-1278

17. Witten, E.: An $S U(2)$ anomaly. Phys. Lett. 117B, 324 (1982)

18. Witten, E.: Talk at the: Symposium on anomalies, geometry and topology.

Bardeen, W.A., White, A.R. (eds.): Global gravitational anomalies. Commun. Math. Phys. 100, 197 (1985)

Communicated by K. Osterwalder

Received June 9, 1986 
